\title{
EFECTOS FISCALES DE LOS ASENTAMIENTOS HIDROELÉCTRICOS: EL CASO DE LA CUENCA DE LOS RÍOS NEGRO Y NARE EN COLOMBIA*
}

\author{
Recibido: 13 de agosto de 2015 • Aprobado: 20 de noviembre de 2015 \\ Juan Pablo Ríos-0campo* \\ Luis Diego Vélez-Gómez ${ }^{* * *}$
}

\section{RESUMEN}

Con la presencia de asentamientos hidroeléctricos en su territorio, los municipios del oriente y nordeste antioqueño han visto afectada su estructura tributaria y territorial, a pesar de la reglamentación compensatoria del sector eléctrico. Este artículo, analiza los efectos tributarios generados por la compensación del impuesto predial. Para lograrlo se revisan los montos recibidos por los municipios de las empresas generadoras y se realiza una indagación empírica del impuesto bajo los escenarios con y sin asentamientos hidroeléctricos. De esta forma se determina el diferencial tributario. Se concluye que, además de la insuficiencia de las transferencias para compensar la pérdida del capital natural de las cuencas hidroeléctricas, la expansión del sector eléctrico genera regresividad tributaria, por causa de privilegios y exenciones concebidos por las Leyes.

\section{PALABRAS CLAVES}

Impuestos locales, impuestos y subsidios, impuestos ambientales y subsidios, externalidades de los impuestos, Colombia.

\section{CLASIFICACIÓN JEL}

H23, H71, R51, R52

\section{CONTENIDO}

Introducción; 1. Embalses, desplazamientos y tributación; 2. Distribución regional de generación hidroeléctrica; 3. Consumo de tierras en la cadena de embalses Río Negro y Nare; 4. Comportamiento del impuesto predial en los municipios con asentamientos hidroeléctricos; 5 . Compensación del predial en la Ley 56/1981; 6. Conclusiones y Recomendaciones; Bibliografía.

Este artículo de investigación es producto del convenio interadministrativo "Acompañamiento y asesoría técnica, ambiental y financiera al Municipio de San Carlos-Antioquia" suscrito entre la Facultad de Minas de la Universidad Nacional de Colombia, Sede Medellín y el municipio de San Carlos, entre los meses de mayo y diciembre de 2013, previo a la construcción del proyecto hidroeléctrico Porvenir II. Fue desarrollado por el grupo de investigación Modelamiento, análisis, energía y economía, clasificación A, Colciencias. Fue financiado en su totalidad por el Municipio de san Carlos.

** Economista, Universidad Nacional de Colombia, Medellín, Colombia. Estudiante de Maestría en Ingeniería de Sistemas, Universidad Nacional de Colombia, Medellín, Colombia. Joven Investigador de Colciencias. Grupo de Investigación Ciencias de la Decisión categoría Al. Dirección de correspondencia: Carrera 80 No. 65-223 Núcleo Robledo, M8A-403. Correo electrónico: jprioso@unal.edu.co.

*** Economista, Universidad de Antioquia, Medellín, Colombia. M.S.C. en Economía del Sector Público, Universidad de Buenos Aires, Instituto Torcuato Di Tella, Buenos Aires, Argentina. Especialista en Finanzas, EAFIT, Medellín, Colombia. Profesor asociado Escuela de Ingeniería de la Organización, Facultad de Minas, Universidad Nacional de Colombia, Sede Medellín. Dirección de correspondencia: Carrera 80 No. 65-223 Núcleo Robledo, M8B-57, teléfono 4255287. Correo electrónico: lvelez@unal.edu.co . 


\title{
FISCAL EFFECTS IN HYDROELECTRIC SETTLEMENTS: THE CASES OF THE RIVERS NEGRO AND NARE BASINS IN COLOMBIA
}

\begin{abstract}
With the presence of hydroelectric settlements in their territory, the municipalities of the eastern and northeastern regions of Antioquia have seen their fiscal and territorial structure affected despite the regulatory compensation established for the electric sector. This article analyses the taxation effects generated by the compensation of property taxes. To do so, different sums received by different municipalities from generating companies are analyzed and an empiric tax analysis is done for both types of territories (with and without hydroelectric settlements). This is how the taxation differential is determined. It is also concluded that, besides the influence of transfers for compensating the loss of the natural capital of the hydroelectric basins, the expansion of the electric sector generates taxation digressivety due to the privileges and exemptions conceived by the law.
\end{abstract}

\section{KEY WORDS}

Local taxes, Taxes and subsidies, Environmental taxes and subsides, tax externalities, Colombia.

\section{JEL CLASSIFICATION}

H23, H71, R51, R52

\section{CONTENT}

Introduction; 1. Reservoirs, displacements and taxation 2. Regional distribution of hydroelectric generation; 3. Land consumption in the reservoir chain of rivers Negro and Nare; 4. Behavior of property tax in the municipalities with hydroelectric settlements; 5 . Property tax compensation in law 56/1981; 6. Conclusions and Recommendations; Bibliography.

\section{EFEITOS FISCAIS DOS ASSENTAMENTOS HIDROELÉCTRICOS: 0 CASO DA BACIA DOS RIOS NEGRO E NARE NA COLÔMBIA}

\section{RESUMO}

Com a presença de assentamentos hidroelétricos no seu território, os municípios do oriente e nordeste antioquenho hão visto afetada sua estrutura tributária e territorial, a pesar da regulamentação compensatória do sector eléctrico. Este artículo, analisa os efeitos tributários gerados pela compensação do imposto predial. Para conseguir se revisam os montantes recebidos pelos municípios das empresas geradoras e se realiza uma indagação empírica do imposto sob os cenários com e sem assentamentos hidroelétricos. Desta forma se determina o diferencial tributário. Se conclui que, ademais da insuficiência das transferências para compensar a perda do capital natural das bacias hidroelétricas, a expansão do setor eléctrico gera regressividade tributária, por causa de privilégios e isenção concebidos pelas Leis.

\section{PALAVRAS CHAVES}

Impostos locais, impostos e subsídios, impostos ambientais e subsídios, externalidades dos impostos, Colômbia.

\section{CLASSIFICAÇÃO JEL}

H23, H71, R51, R52

\section{CONTEÚDO}

Introdução; 1. Embalses, deslocamentos e tributação; 2. Distribuição regional de geração hidroelétrica; 3. Consumo de terras na cadeia de barragens Rio Negro e Nare; 4. Comportamento do imposto predial (IPTU) nos municípios com assentamentos hidroelétricos; 5. Compensação do IPTU na Lei 56/1981; 6. Conclusões e Recomendações; Bibliografia. 


\section{INTRODUCCIÓN}

La trascendencia del sector eléctrico en el desarrollo económico de un país conlleva a que la planeación y el proceso de licenciamiento de los proyectos sean prioridad en la toma de decisiones por parte de los gobiernos nacionales, dado que en ningún otro sector como en este, la importancia de los servicios trasciende el valor económico para poner de presente el valor estratégico de los mismos, como lo expresan Vélez y otros (1982).

En tal circunstancia, los asentamientos hidroeléctricos (AH) en casi todos los países del mundo obedecen a una política de seguridad nacional. Esto significa que las autorizaciones y licencias de los proyectos se toman de manera central y casi siempre chocan con las visiones territoriales e identidades regionales, las cuales son fracturadas cuando se implementa una obra de este tipo, tal y como lo mencionan Dourojeanni, Jouravlev y Chávez (2002)

Los impactos económicos de un proyecto hidroeléctrico afectan de manera directa la estructura tributaria de los municipios en dos formas: por los terrenos destinados al embalse, porque desaparece toda actividad económica allí localizada (agricultura, pesca, minería y pequeña ganadería); y, aguas abajo del embalse, porque los cambios en las condiciones del agua modifican los parámetros de producción y perjudican los sectores económicos relacionados con el río, como lo argumenta Acosta (2004).

En este aspecto, las acciones de reparación se fijan sobre las negociaciones de los títulos de propiedad de las tierras, al sacrificar los bienes comunes (aguas, tierras, bosques), y servicios ecosistémicos del capital natural de la región y de los municipios. Estos bienes pasan a servir en forma intensiva en la generación de energía, en perjuicio del patrimonio ambiental, fiscal y cultural de los municipios sin que medien acciones de resarcimiento debidamente valoradas, de acuerdo a Dourojeanni, Jouravlev y Chávez (2002).

Al tener en cuenta que el aumento de las rentas energéticas es inversamente proporcional a la participación que tienen los municipios en ellas, se observa que los AH han generado un efecto regresivo en la tributación municipal. Esto se afirma porque antes de la implementación de los proyectos los municipios recibían tributos por tierras, edificaciones e inmuebles, los que, en virtud del tratamiento tributario privilegiado que concedió la Ley 56 del Congreso de Colombia (1981) a la expansión del sector eléctrico, son sacrificados con las exenciones y descuentos consignados en el artículo 4to de esta Ley. 
Por lo tanto, este artículo se propone en primer lugar evaluar el nivel de sacrificio de los impuestos locales en los municipios del Oriente y el Nordeste antioqueño por efecto de la presencia de asentamientos hidroeléctricos. Por medio del análisis de las compensaciones del impuesto predial, se valoran los efectos tributarios sobre la estructura fiscal y productiva de los municipios bajo estudio. Para finalizar, se plantea una discusión sobre la regresividad fiscal producida en los municipios en razón al tratamiento preferencial a las empresas generadoras de hidroelectricidad.

\section{EMBALSES, DESPLAZAMIENTOS Y TRIBUTACIÓN}

De acuerdo a la Comisión Mundial de Represas (2000), se estima que las mega-obras hidroeléctricas son responsables del 63\% de los desplazamientos poblacionales, por causas ambientales. Para Colombia, Martínez (2013) calcula que la población desplazada, por el 30\% de la capacidad eléctrica, representó un total de 23.675 personas, pertenecientes a minorías, campesinas, afro-descendientes e indígenas. Según Larrahondo (1993), la cuenca del río Magdalena, la cual ocupa una superficie de 260,000 km² equivalente al 22\% del territorio del país y al 70\% de la población, recibió los mayores impactos debido a la intensidad poblacional y al número de asentamientos localizados allí.

La zona Caribe, más intensiva en centrales térmicas y a gas, resulta afectada por causa de la represa Urra I, abastecida por el caudaloso río Sinú, la cual desplazó a 12.000 personas y afectó a 60.000 pescadores aguas abajo que perdieron, sin una clara compensación económica, la fuente primordial de su subsistencia, de acuerdo a la Comisión Mundial de Represas (2000, p. 115-116). No obstante, todavía está latente la construcción de Urra II con una incorporación nueva de 50.000 hectáreas.

Guhl (2014) explica este panorama como una consecuencia directa de una planificación centralizada que defiende intereses de entidades internacionales, en los que la "diversidad natural y cultural de sus regiones" es dejada de lado. Además, resalta la poca legitimidad de las políticas ambientales, al anotar que más allá de la formulación aparece la falta de efectividad en la planificación y gestión ambiental, lo cual se considera un freno para el desarrollo, tal como lo menciona Torres (2013).

En este sentido, cabe preguntarse entonces si la explotación de los abundantes recursos hidroenergéticos del país ha permitido influir de manera positiva en la actual configuración de la estructura fiscal de los municipios con asentamientos, y si se han inducido modificaciones tributarias que permitan caracterizarla como más dinámica y diversa. Desafortunadamente, la respuesta que se pretende dar es 
Efectos fiscales de los asentamientos hidroeléctricos: el caso de la cuenca de los ríos Negro y Nare en Colombia

negativa, porque la evidencia muestra, en la parte fiscal, un efecto empobrecedor sobre las bases tributarias de los municipios que conforman los embalses y las cuencas hidroeléctricas.

En medio de estas contradicciones legales y sociales, los AH han crecido de manera acelerada al inundar grandes territorios que, en otro tiempo, fueron regiones para la agricultura, la pesca, la minería y la ganadería, como lo muestran Ansar y otros (2014). Como resultado, la salida de tierras de la tributación local se debe en mucho a las deficiencias en la legislación por parte del Estado; dado no solo el enorme rezago en la valoración de los predios rurales, sino también las ventajas tarifarias concedidas a las generadoras, a pesar de que la tributación subnacional representa menos del 15\% del recaudo total y el 2,9\% del PIB, tal y como lo menciona Fuentes (2013).

Más aun, de acuerdo a la evaluación de los informes realizada por la OCDE y CEPAL (2014, p. 87) para el ingreso del país a la organización, se concluye que la mayoría de gravámenes ambientales son originados en la explotación de recursos no renovables (combustibles), observándose que no hay ninguno dirigido a fuentes renovables como la hidroelectricidad. Este argumento se sostiene porque, a pesar de un incremento de cerca de 30\% entre 2000 y 2011 de los impuesto ambientales, estos apenas representaban el $0.7 \%$ del PIB y el $3.7 \%^{1}$ de la tributación total del país.

Esta situación se genera porque en virtud de la primera Ley que estableció compensaciones a los municipios con asentamientos hidroeléctricos, Ley 56/1981, se aplican hoy a las empresas del sector hidroeléctrico, tarifas y avalúos preferenciales. Además, el Decreto 2024/1982, Congreso de Colombia (1982), que reglamentó la Ley, estipula que el impuesto predial vigente es la tarifa que regía el 5 de octubre de 1981 para las obras construidas o en construcción, y también será la tarifa que rija a la fecha de la construcción de un nuevo proyecto. El efecto subsiguiente fue la congelación de las tarifas en el tiempo.

De la misma forma, para los avalúos de las propiedades permanentes se indicó en el mismo Congreso de Colombia (1981) que no se tendrán en cuenta, para el cálculo del impuesto predial a pagar, las construcciones asociadas a la generación de electricidad. Así mismo, el área cubierta por el embalse, que es una parte significativa de los territorios municipales, queda exenta de impuesto, ya que no existe un tributo compensatorio sobre el espejo de agua que cubre los embalses.

1 En promedio, en los países agrupados en la OCDE, los impuestos ambientales representan el 1.6\% del PIB y el 5.6\% dentro de los ingresos tributarios. 
Por otra parte, el Artículo 45 de la Ley 99/1993, Congreso de Colombia (1993), contempla que es deber del sector eléctrico transferir el $6 \%$ de las ventas brutas de energía por generación propia, conforme a la tarifa que para venta en bloque de energía señale la Comisión Reguladora de Gas y Energía (CREG). Por ello, se argumenta que en sustitución a las anteriores exenciones están las transferencias consagradas en la misma Ley y que el porcentaje del 3\% de las ventas de energía generada en bloque estaría dirigido a compensar a los municipios con cuencas hidroeléctricas.

En esta dirección, la distribución del anterior porcentaje contempló para la asignación los criterios de participación en la cuenca hidroeléctrica (1.5\%) y la proporción del embalse perteneciente al municipio (1.5\%). No obstante, esta distribución tiene efectos fiscales negativos, porque el criterio determinante para fijar la cantidad de transferencias entre municipios es el de "capacidad instalada", en perjuicio del criterio "tamaño de embalse" que no solo es más demandante de tierras sino que tambien tiene efectos debastadores, tanto del patrimonio ambiental de los territorios como de la estructura fiscal, tal como lo mencionan Ansar y otros (2014).

El detrimento fiscal, respecto a la Ley 56/1981, está asociado a las zonas de embalse, que son las que aparecen excluidas de la tributación local. Al final, este ultimo criterio es el elemento más visible del aporte de los municipios a los AH.

\section{DISTRIBUCIÓN REGIONAL DE GENERACIÓN HIDROELÉCTRICA}

La energía hidráulica es la principal fuente de generación de energía eléctrica del país con una participación cercana al 70\%, registrada en el Sistema Interconectado Nacional. Esta energía proviene de 21 embalses y 18 centrales distribuidas en cinco regiones hidrológicas. De estas regiones, Antioquia aporta el 44,6\% de la energía almacenada, seguida por la región Centro con 32\%, Oriente y Valle con 18.8\% y Caribe con 4,6\%, tal como lo reporta XM (2014), ver gráfico 1.

Con 3.757,51 megavatios (MW) de capacidad instalada, Antioquia representa la tercera parte de la capacidad de generación de energía hidroeléctrica del país (ver tabla 1), a pesar de que esta cifra solo representa el $16 \%$ de la capacidad total del departamento, 23.556 MW. Por tanto, Antioquia cuenta con un potencial por explotar de 19.798 MW. Con esta participación, que equivale a 46 centrales hidroeléctricas construidas desde 1947 y de las cuales 25 pertenecen a EPM, se puede afirmar que la zona antioqueña ha aprovechado la ventaja comparativa de su hidrología por medio de un consolidado clúster empresarial, el cual incluye capacidades tecnológicas derivadas de sus tradicionales centros académicos, como muestra BIRD Antioquia (2011). 
Efectos fiscales de los asentamientos hidroeléctricos: el caso de la cuenca de los ríos Negro y Nare en Colombia

Gráfico 1. Energía almacenada en embalses por región hidrológica en 2014.

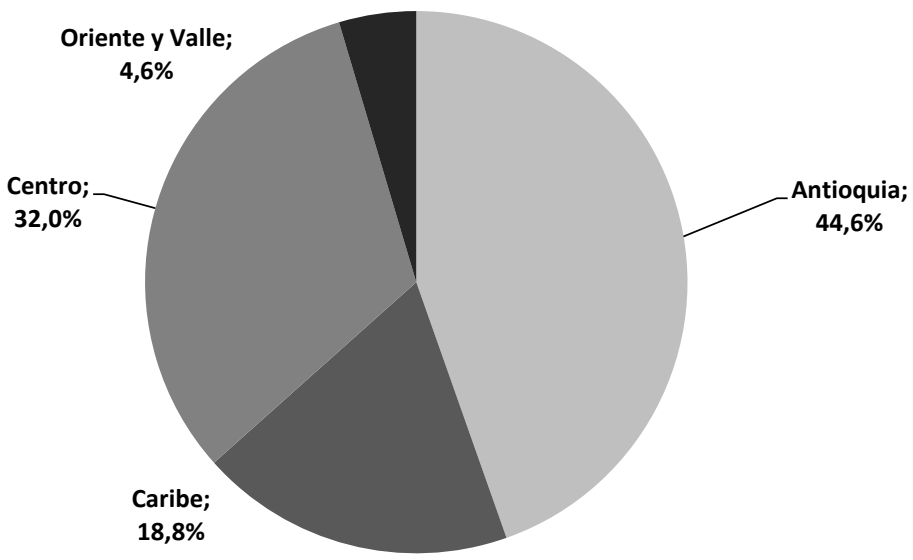

Fuente: elaboración propia con información de XM (2014)

Tabla 1. Potencial energético de Antioquia

\begin{tabular}{|c|c|c|}
\hline Entidad & Capacidad Instalada MW & Participación (\%) \\
\hline EPM & $2.203,70$ & 58.64 \\
\hline ISAGEN & 1.436 & 38.21 \\
\hline Otros & 117,81 & 3.13 \\
\hline Total & $3.757,51$ & 100 \\
\hline
\end{tabular}

Fuente: elaboración propia con información de BIRDAntioquia (2011)

En forma adicional, hoy se encuentran en etapa de construcción seis proyectos hidroeléctricos que le darán al departamento, y al país, una capacidad instalada adicional de $3.791 \mathrm{MW}$, configurándose el proyecto Hidro-Ituango como el más grande de la región y del país, el cual aportará 2.400 MW de capacidad instalada al sistema interconectado (ver tabla 2).

De esta manera, el departamento de Antioquia se ha convertido en el eje de la programación hidroeléctrica del país donde dos subregiones -el oriente y nordesteaportan el 75\% de la capacidad instalada de todo el departamento. Así mismo, de los cinco embalses con mayor capacidad de almacenamiento de estas subregiones, cuatro de ellos están ubicados en la subregión del oriente que ocupan un área aproximada de 13.500 hectáreas (ha) en una sola cadena que inicia en El Peñol y se extiende por los municipios de Alejandría, Granada, Guatapé, Santo Domingo, San Rafael, San Roque y San Carlos hasta el norte del departamento de Caldas. Ello 
sin tomar en cuenta la cadena del río Samaná, donde el solo embalse del proyecto Porvenir II contará con 75 km de longitud.

Tabla 2. Proyección hidroeléctrica

\begin{tabular}{|l|c|c|}
\hline \multicolumn{1}{|c|}{ Central } & $\begin{array}{c}\text { Capacidad efectiva } \\
\text { bruta(MW) }\end{array}$ & Propietario \\
\hline Hidro-Ituango & 2.400 & EPM \\
\hline Sogamoso & 800 & ISAGEN \\
\hline El Quimbo & 396 & ISAGEN \\
\hline Cucuana & 60 & EPSA \\
\hline Miel II & 135 & EPSA y GENSA \\
\hline Total & 3.791 & - \\
\hline
\end{tabular}

Fuente: elaboración propia con información de BIRD Antioquia (2011)

En tal circunstancia, la conformación del sector hidroeléctrico en las subregiones de Antioquia permite fijar el objeto de análisis que corresponde al examen de los impactos fiscales de las centrales hidroeléctricas en las subregiones de oriente y nordeste antioqueño,

\section{CONSUMO DE TIERRAS EN LA CADENA DE EMBALSES RÍO NEGRO Y NARE}

Como se observa, los territorios del oriente antioqueño están ocupados hoy por las mayores centrales del departamento y del país. Estas centrales son: Peñol-Guatapé (en El Peñol y Guatapé), Jaguas (en San Rafael), Playas (en San Rafael y San Carlos), Punchiná (en San Carlos) y Calderas (en Granada) (ver mapa 1). En total, los embalses de estos proyectos demandan un volumen de almacenamiento de 1.596 millones $\mathrm{m}^{3}$.

Respecto al consumo de tierras, por orden de afectación y participación en hectáreas dentro de los municipios, la central Peñol-Guatapé ${ }^{2}$ se ubica como la de mayor impacto por hectáreas inundadas, seguida por los sistemas Jaguas, Playas, Punchiná y Calderas -ver Tabla 3. Estas centrales hidroeléctricas afectan, por la sola ubicación de los embalses ocho municipios, los cuales han sacrificado para la expansión hidroeléctrica su capital natural, su estructura productiva y su base tributaria.

2 Otros municipios afectados son Marinilla (9 ha), Concepción (49 ha) y San Vicente (25 ha). 
Mapa 1. Cadena de embalses del nordeste y oriente antioqueño

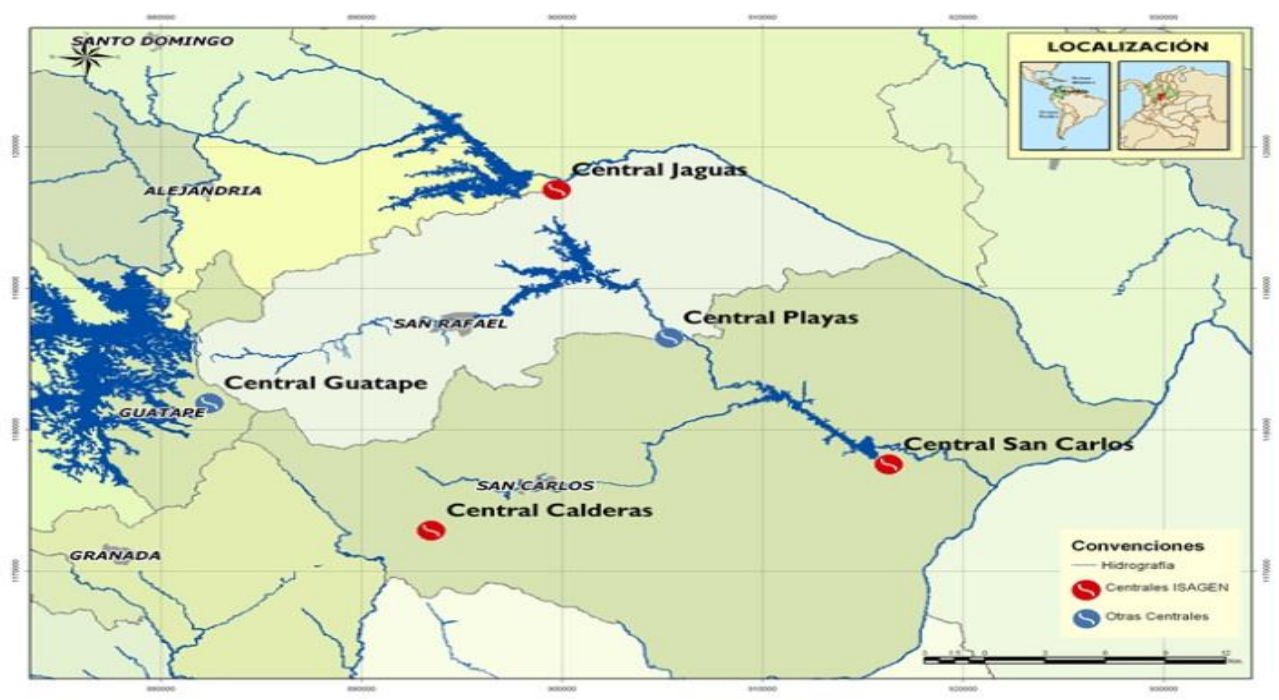

Fuente: Tomado de ISAGEN

Tabla 3. Municipios con influencia de proyectos hidroeléctricos

\begin{tabular}{|l|c|c|}
\hline \multicolumn{1}{|c|}{ Proyecto hidroeléctrico } & Municipio & Hectáreas embalse \\
\hline \multirow{2}{*}{ Punchiná } & San Carlos & 366 \\
\cline { 2 - 3 } & Granada & 12 \\
\hline \multirow{4}{*}{ Playas } & San Rafael & 702 \\
\hline \multirow{4}{*}{ Peñol-Guatapé } & El Peñol & 2.936 \\
\cline { 2 - 3 } & Guatapé & 3.017 \\
\cline { 2 - 3 } & Alejandría & 329 \\
\hline \multirow{3}{*}{ Jaguas } & San Roque & 197,4 \\
\cline { 2 - 3 } & Alejandría & 760,4 \\
\cline { 2 - 3 } & Santo Domingo & 72,4 \\
\hline \multirow{2}{*}{ Calderas } & Granada & 4 \\
\cline { 2 - 3 } & San Carlos & 4 \\
\hline
\end{tabular}

Fuente: elaboración propia con información de la Resolución 686/1994, Gobernación de Antioquia (1994), y la Dirección de sistemas de información y catastro de Antioquia.

La expansión hidroeléctrica, a la que se sumarán más de 57 microcentrales en estudio y/o con licencia ambiental otorgadas por las Corporaciones Regionales en estas subregiones, se ha convertido en un motivo legítimo de alarma ante el enorme consumo de tierras y el consecuente sacrificio tributario que implica una central hidroeléctrica. 
La razón principal de este sacrificio fiscal se debe a que los terrenos ubicados en el área de influencia de un proyecto hidroeléctrico soportan tarifas preferenciales del impuesto predial -que no varían- desde la expedición de la Ley 56/1981 y el Decreto reglamentario 2024/1982, máxime cuando la misma legislación excluye del pago a las presas, estaciones generadoras y, en general, al área del embalse; ello; sin establecer hasta el momento un tributo compensatorio para el espejo de agua que cubre extensas cantidades de tierras municipales. Estos son los determinantes más fuertes para explicar el acelerado proceso de empobrecimiento tributario en los municipios en Antioquia con proyecto hidroeléctricos.

El caso del embalse Peñol-Guatapé, de propiedad de las EPM, en los municipios de Guatapé y El Peñol, es un típico ejemplo de los cambios sociales, económicos, ambientales y territoriales producidos por causa de este proyecto hidroeléctrico. Para disponer del embalse que cuenta con una capacidad de 1.169 millones de $\mathrm{m}^{3}$, fueron desplazados 7.550 habitantes y se sacrificó casi el 60\% del municipio de Guatapé según Acosta (2004) y todo el casco urbano del Peñol tal y como lo mencionan la Alcaldía de El Peñol (2014) y Torres (2013).

Como efecto del nuevo uso territorial y dado la cercanía con el espejo de agua, Guatapé asumió una vocación dirigida hacia el turismo, conforme describen Aramburo y Carmona (1990). Aun así, esta nueva vocación económica, antes que mejorar la situación fiscal del municipio, ha incrementado los costos de inversión en salud, seguridad, saneamiento ambiental y mantenimiento de vías.

En la actualidad, Guatapé sufre afectaciones tanto por la pérdida del impuesto predial como por el efecto de los predios exentos del pago del impuesto (presa, estaciones generadoras y otras obras públicas), que en conjunto representan el $6 \%$ del área total del municipio. Esta área se magnificó luego, porque por medio de ella se vincula el área del embalse, con lo cual EPM ha obtenido un beneficio correspondiente al 47,4\% del municipio, a partir de la Ley 56/1981, o lo que es lo mismo; el conjunto el área total afectada está cerca del 53\% del territorio municipal.

Al respecto, Catastro Departamental (2013) reporta que para la misma cuenca, otros municipios que resultan afectados en términos territoriales por la cadena de embalses son Alejandría en 24,83\%, El Peñol en 24,58\%, San Rafael en 12,86\% y Concepción en $7,49 \%$.

Un caso especial es San Carlos, el cual aparece como el municipio más intensivo en la utilización de recursos hidroeléctricos, dado que en su territorio está instalado el 97\% del embalse de Punchiná, un 44\% del embalse de Calderas y un 1.13\% de la cuenca de la central de Playas (366 ha). Tiene además comprometido parte del 
Efectos fiscales de los asentamientos hidroeléctricos: el caso de la cuenca de los ríos Negro y Nare en Colombia

territorio, junto a San Luis y Puerto Nare, para el embalse del proyecto Porvenir II, el cual requiere la inundación de 17 localidades, respecto al estudio realizado por PROESA (2013).

Actualmente, por efecto de la sedimentación, la colmatación y el cambio climático que ha mermado la vida útil de los embalses, se percibe la alteración de las condiciones naturales iniciales, lo cual limita las posibilidades de la explotación económica por parte de las comunidades locales, ya que el embalse se convierte en un terreno perdido para siempre, como bien dice el campesinado de la región.

\section{COMPORTAMIENTO DEL IMPUESTO PREDIAL EN LOS MUNICIPIOS CON ASENTAMIENTOS HIDROELÉCTRICOS}

De acuerdo a Jorratt (2010, p. 73), el impuesto predial tiene características que lo ubican como la mejor fuente de financiamiento local, dado que se aplica sobre un activo inamovible, es decir, inelástico. También, porque induce al uso productivo de los activos, al facilitar el recaudo y favorecer la actividad agrícola y el mercado inmobiliario. Es, así mismo, un instrumento de equidad, dado que las tarifas se deberían cobrar en función del valor de la propiedad comercial.

Del mismo modo, Cárdenas y Mercer-Blackman (2005, p. 17) argumentan que este es un impuesto "simple, transparente y fácil de recaudar y administrar, lo [cual] (...) hace casi imposible la evasión"; además de representar una fuente que, por ser de libre destinación, sirve para que los municipios ejerzan autonomía financiera.

No obstante, al respecto Iregui, Melo y Ramos $(2004$, p. 1) argumentan que "los recaudos por predial son inferiores a su potencial, debido al rezago en la actualización de los avalúos catastrales, al bajo nivel de las tarifas nominales y a la existencia de una brecha entre las tarifas nominales y efectivas". Esa situación se presenta en aquellos municipios pequeños y medianos, según Naranjo y otros (2011), en los cuales la baja capacidad de recaudo tributario conecta con una alta dependencia de las transferencias del gobierno, de acuerdo a Bonet (2006), y con una ausencia marcada de autoridad catastral competente, tal y como lo manifiesta Nuñez (2005, p. 29). Un dato ilustrativo de esta situación muestra que en Colombia el 33\% de los municipios presentan rezago catastral; pero en Antioquia la cifra llega al 88,9\% de los municipios con AH, sobre todo en el área rural, conforme a las cifras reportadas por el DNP (2013).

De esta manera, el comportamiento que ha presentado el impuesto predial en los municipios del oriente y el nordeste antioqueño refleja una tendencia decreciente en la participación dentro del conjunto de la estructura tributaria local (ver gráfico 2). Mientras que en el conjunto de municipios del país el impuesto predial tiene 
una participación del 33,1\%, como lo indica el DNP (2014a); dentro de los ingresos tributarios, en los municipios con AH su participación tiende hacia el 30\%. Entre el 2000-2012, los municipios con mayor disminución en la participación fueron Santo Domingo (-26\%), San Roque (-25\%) y Concepción (-25\%); municipios con afectaciones hidroeléctricas.

Gráfico 2. Participación del impuesto predial en los ingresos tributarios

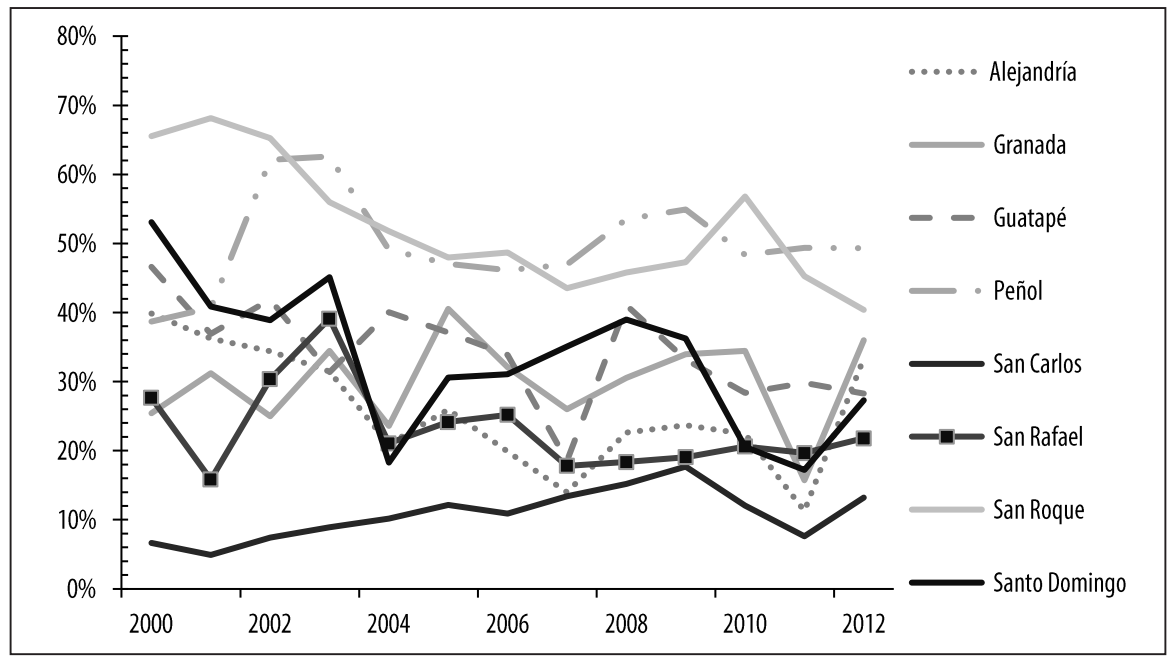

Fuente: elaboración propia con información de DNP (2014b),

Otro frente de controversia fiscal que sigue sin resolverse esta suscitado por el Congreso de Colombia (2012) que exige a las empresas generadoras de energía eléctrica aplicar el Artículo $7^{\text {to }}$ de la Ley 56/1981³, el cual establece que se debe gravar a las empresas sobre la base de su capacidad instalada solo bajo la ubicación donde se desarrollan las actividades industriales. Sin embargo, Nuñez (2005) manifiesta que como efecto sustitución, se ha presentado un mayor dinamismo en los impuestos de industria y comercio y en la sobretasa a la gasolina ello como resultado de la proliferación de nuevos negocios compatibles con el asentamiento hidroeléctrico (cantinas, estaciones de gasolina, restaurantes y, en general, negocios del sector terciario). Sobre este punto Fuentes (2013) define este proceso como un recurso de protección fiscal por parte de los municipios

A este respecto hay que advertir que los problemas de interpretación sobre conceptos como servicio público domiciliario, operador del servicio y otros atajos jurisprudenciales han impedido que el Artículo $7^{\text {to }}$ de la Ley 56/1981 quede en

3 Según este artículo, las entidades propietarias de los asentamientos hidroeléctricos podrán ser gravadas con el impuesto de industria y comercio, hasta \$5/KW anuales, que se reajusta con el IPC cada año, por cada central generadora. 
Efectos fiscales de los asentamientos hidroeléctricos: el caso de la cuenca de los ríos Negro y Nare en Colombia

vigencia, todo lo cual perjudica, aún más, las golpeadas finanzas de los municipios afectados por obras hidroeléctricas. Esto significa que el impuesto de industria y comercio de la actividad generadora es otro asunto de evasión fiscal en la actividad generadora.

\section{COMPENSACIÓN DEL PREDIAL EN LA LEY 56/1981}

La compensación del impuesto predial, establecida en el Artículo $4^{\text {to }}$ de la Ley 56/1981, surge como medida para contrarrestar el sacrificio tributario causado por el uso de los terrenos en los proyectos hidroeléctricos. La compensación busca que las empresas ejecutoras de los proyectos hidroeléctricos paguen los recursos que los municipios afectados dejan de percibir vía impuesto predial. Esta compensación se justificó, porque antes de 1981 los municipios no recibían ninguna reposición por los predios inundados, como lo expone la Gobernación de Antioquia (1985).

No obstante, los lineamientos para llevar a cabo el pago del impuesto y la compensación del predial por las empresas generadoras, el efecto fiscal ha sido contrario. Ello, porque lo estipulado en la Ley desalentó el recaudo potencial del impuesto predial en razón a las exenciones, al carácter preferencial concedido a las empresas generadoras en las tarifas del impuesto predial y a la desactualización de los avalúos.

Lo anterior se evidencia en el literal b) del Artículo $4^{\text {to }}$ de la Ley 56/1981; en el cual se establece que las empresas generadoras deben pagar "por los edificios y por las viviendas permanentes de su propiedad"; sin embargo, se excluyen del pago las presas, estaciones generadoras y otras obras públicas con los equipos. De otra parte, en el parágrafo se determina pagar el 150\% de la tasa que "corresponde al impuesto predial vigente para todos los predios en el municipio". Tasa que sería "congelada" en el Decreto 2024/1982 como aquella que se encontraba vigente el 5 de octubre de 1981.

Así entonces, aunque la Ley se vio en su momento como un logro tributario por parte de los municipios, este logro quedó mermado porque las empresas de energía asumieron tarifas y avalúos preferenciales que afectaron no solo la eficiencia del tributo, sino también la equidad. Esto se evidencia porque hoy un habitante promedio de los municipios del oriente y del nordeste antioqueño paga un impuesto predial más alto que cualquier empresa generadora (ver tabla 4).

En síntesis, las pérdidas tributarias se explican por i) la regresividad tributaria asociada al anclaje de las tarifas para el pago de la compensación del impuesto predial y ii) por las distorsiones en la información utilizada para el cálculo de la compensación del predial conforme al parágrafo del Artículo $4^{\text {to }}$ de la Ley 56/1981. 


\subsection{Regresividad tributaria}

Con la reglamentación de la Ley 56/1981, los proyectos hidroeléctricos que entraron en funcionamiento antes de ese año, y aquellos que entraron en los años siguientes, se vieron obligados a compensar, con la tarifa local de la época, a los municipios afectados por los predios inundados. No obstante, esta compensación no produjo los efectos deseados debido a los descuentos tarifarios y a las exenciones que permitió esta Ley (ver tabla 4).

Esto represento un desajuste fiscal en el tiempo para los municipios, ya que a medida que se actualizaba el estatuto tributario, los impuestos aumentaban para los habitantes y demás sectores productivos, pero no para las empresas generadoras. El resultado fue que los pagos, por las generadoras hidroeléctricas, fueron inferiores a las otras actividades económicas y a los habitantes. De esta manera, la preferencia tarifaria de las empresas generadoras quedó ligada a la vida promedio de los proyectos, que de por si es mayor a 40 años de vida útil.

En la tabla 4, se presentan las disparidades en la tarifa del impuesto predial que rige para los diferentes sectores productivos y para la población, tanto urbana como rural, en los municipios con presencia de AH del Oriente y Nordeste antioqueño. En San Carlos la diferencia entre las tarifas cobradas a las empresas generadores de energía y a los habitantes es del $45 \%$.

Tabla 4.Tarifas municipales del impuesto predial por sectores

\begin{tabular}{|l|c|c|c|c|c|}
\hline \multicolumn{1}{|c|}{ Municipio } & Ley 56/1981 & $\begin{array}{c}\text { Sectores produc- } \\
\text { tivos rural }\end{array}$ & $\begin{array}{c}\text { Sectores produc- } \\
\text { tivos urbano }\end{array}$ & $\begin{array}{c}\text { Residentes }(r) y \\
\text { agropecuarios }(a) \\
\text { rural }\end{array}$ & $\begin{array}{c}\text { Residentes } \\
\text { urbano }\end{array}$ \\
\hline San Carlos & 6 & $7-15$ & $8-16$ & $6-15$ & $7-16$ \\
\hline Alejandría & 6 & 10 & 10 & $7(\mathrm{r})-8(\mathrm{a})$ & 8 \\
\hline Granada & 9 & 8 & 8 & 6 & 7 \\
\hline Santo Domingo & 6 & $10-16$ & $8-11$ & $4-11(\mathrm{a})$ & $5-11$ \\
\hline El Peñol & 6 & $7-12$ & $8-12$ & $4(\mathrm{a})$ & $6-10$ \\
\hline San Roque & 6 & $11,5-14,5$ & 11,5 & $7,5(\mathrm{a})$ & $9,5-15$ \\
\hline Guatapé & 6 & $6-8$ & $6-8$ & 5 & $4-6$ \\
\hline
\end{tabular}

Valores por mil

Fuente: elaboración propia con información de los Acuerdos Municipales ${ }^{4}$, ISAGEN y Gobernación de Antioquia (1985).

4 En San Carlos rige el Acuerdo 19/2005; para Alejandría, el Acuerdo 013/2004; para Granada, el Acuerdo 15/2004; para Santo Domingo, el Acuerdo 15/2010; para El Peñol, el Acuerdo 016/2006; para San Roque, el Acuerdo 014/2006; para Guatapé, el Acuerdo 14/2013. 
Resulta incomprensible entonces que las generadoras paguen tarifas inversamente proporcionales a la rentabilidad del activo, la cual se registra entre el 10.4\% y 14.4\%. Esto se ilustra en los municipios del Peñol y Guatapé, los cuales contienen más de 6.800 hectáreas del embalse de la central Peñol-Guatapé. Allí las tasas oscilan entre el 8 y el 12 por mil.

Sin embargo, Catastro Departamental (2013) evidencia que la desigualdad tributaria es mayor al observar que, para el caso de Guatapé, el sector eléctrico, representado por EPM, tiene afectada más de la mitad del territorio municipal; razón por la cual el bajo nivel de las tarifas genera una alta inequidad respecto a los demás contribuyentes. Máxime si se tiene en cuenta que el territorio residual, después del embalse, no alcanza para recuperar el potencial tributario que tendría sin la presencia de la central hidroeléctrica.

\subsubsection{Perdida tributaria}

Para determinar cuántos recursos son dejados de percibir por los municipios debido a la presencia de los AH, se determina el diferencial entre el impuesto predial reportado, de acuerdo a la compensación pagada (tabla 5), y la eventual recaudación predial sin contar con predios afectados por las hidroeléctricas, los cuales se gravarían con la tarifa regular del 10x1000. Esta cuantificación obliga al examen de los montos transferidos, por las empresas generadoras, para compensar a los municipios.

Tabla 5. Compensación pagada del impuesto predial

\begin{tabular}{|c|c|c|c|c|c|c|c|c|}
\hline Año & Alejandría & El Peñol* & Guatapé $^{*}$ & San Carlos & San Rafael* & San Roque & $\begin{array}{c}\text { Santo } \\
\text { Domingo }\end{array}$ & Granada \\
\hline 2009 & 8,72 & 234,89 & 192,33 & 65,44 & 25,33 & 3,13 & 1,78 & 0,19 \\
\hline 2010 & 7,59 & 237,53 & 200,26 & 65,98 & 22,26 & 3,19 & 1,81 & 0,18 \\
\hline 2011 & 7,53 & 237,94 & 203,47 & 67,01 & 23,19 & 3,19 & 1,83 & 0,18 \\
\hline 2012 & 7,52 & 241,13 & 209,12 & 71,48 & 23,15 & 3,15 & 1,89 & 0,18 \\
\hline 2013 & 14,57 & 233,39 & 209,98 & 70,78 & 21,20 & 3,16 & 1,91 & 0,18 \\
\hline
\end{tabular}

* Las cifras del 2013 fueron estimadas con el promedio de los últimos 5 años.

Cifras en millones de pesos.

Fuente: elaboración propia con información de ISAGEN y EPM.

En la tabla 6 se observa que el diferencial obtenido en los municipios de El Peñol, Guatapé y San Carlos durante 2010-2013, son negativos, lo cual indica el detrimento tributario que enfrentan los municipios con la presencia de hidroeléctricas en su jurisdicción. 
Tabla 6. Pérdida tributaria 2010-2013 con y sin AH

\begin{tabular}{|c|c|c|c|c|c|c|c|c|c|}
\hline & \multicolumn{3}{|c|}{ El Peñol* } & \multicolumn{3}{c|}{ Guatapé* $^{*}$} & \multicolumn{3}{c|}{ San Carlos } \\
\hline Año & $\begin{array}{c}\text { Con AH } \\
\text { 6 por mil }\end{array}$ & $\begin{array}{c}\text { Sin AH } \\
10 \text { por mil }\end{array}$ & $\begin{array}{c}\text { Pérdida } \\
\text { tributaria }\end{array}$ & $\begin{array}{c}\text { Con AH } \\
6 \text { por mil }\end{array}$ & $\begin{array}{c}\text { Sin AH } \\
10 \text { por mil }\end{array}$ & $\begin{array}{c}\text { Pérdida } \\
\text { tributaria }\end{array}$ & $\begin{array}{c}\text { Con AH } \\
6 \text { por mil }\end{array}$ & $\begin{array}{c}\text { Sin AH } \\
10 \text { por mil }\end{array}$ & $\begin{array}{c}\text { Pérdida } \\
\text { tributaria }\end{array}$ \\
\hline 2010 & 1106 & 1587 & -481 & 570 & 695 & -124 & 333 & 484 & -151 \\
\hline 2011 & 1147 & 1628 & -481 & 554 & 679 & -126 & 230 & 381 & -151 \\
\hline 2012 & 1195 & 1675 & -481 & 650 & 765 & -115 & 426 & 621 & -195 \\
\hline 2013 & 1351 & 1870 & -519 & 657 & 787 & -130 & 453 & 653 & -200 \\
\hline
\end{tabular}

* Las cifras para el 2013 fueron estimas con promedio de los últimos cinco años.

Cifras en millones de pesos

Fuente: elaboración propia con información del DNP (2014b).

La pérdida tributaria por concepto de impuesto predial ocasionada por la presencia de AH, representa, en promedio, en San Carlos cerca de \$174 millones entre el 2010-2013. El Peñol, en igual periodo, presenta una pérdida tributaria mayor, por efecto de mayor número de predios inundados. Bajo este escenario, se deja de percibir en promedio al año $\$ 490$ millones. Mientras tanto, para Guatapé la pérdida tributaria alcanza a \$124 millones anuales.

En el gráfico 3, se muestran las pérdidas tributarias para los municipios bajo estudio. Allí, la distancia entre las dos líneas, deja ver la enorme brecha que separa los comportamientos fiscales de los municipios con AH, de los municipios no afectados con asentamientos. La línea gris representa el recaudo, vía impuesto predial, que los municipios recibirían en presencia de los tradicionales sectores productivos (sin AH). Por su parte, la línea negra representa el recaudo vía impuesto predial reportado por los municipios al Departamento Nacional de Planeación, el cual incorpora las compensaciones pagadas por los $\mathrm{AH}$ al reemplazar la tributación de los sectores productivos.

Se observa que, aunque la Ley 56/1981 determinó que las empresas generadoras debían compensar a los municipios por los predios inundados, las compensaciones realizadas no han logrado resarcir los impactos tributarios producidos por los $\mathrm{AH}$ en los municipios que perdieron las vocaciones tradicionales.

Así mismo, se concluye entonces que las pretensiones de la Ley 56/1981 de compensar el impuesto predial dejado de percibir por los municipios, por cuenta del territorio afectado, en realidad lo que produjo fue una perdida tributaria. Esto se ha evidenciado por el diferencial tributario hallado entre el escenario fiscal de las actividades tradicionales, sin $\mathrm{AH}$, versus el escenario fiscal con $\mathrm{AH}$. Allí se muestra 
que lo pagado por las empresas generadoras a los municipios, según lo establece el Artículo $4^{\text {to }}$ de la Ley 56/1981, es mucho menor que la captación tributaria en ausencia de las hidroeléctricas, lo cual genera un fallo de asignación contenido en el criterio de aplicación de la Ley.

\section{Gráfico 3. Pérdida tributaria}

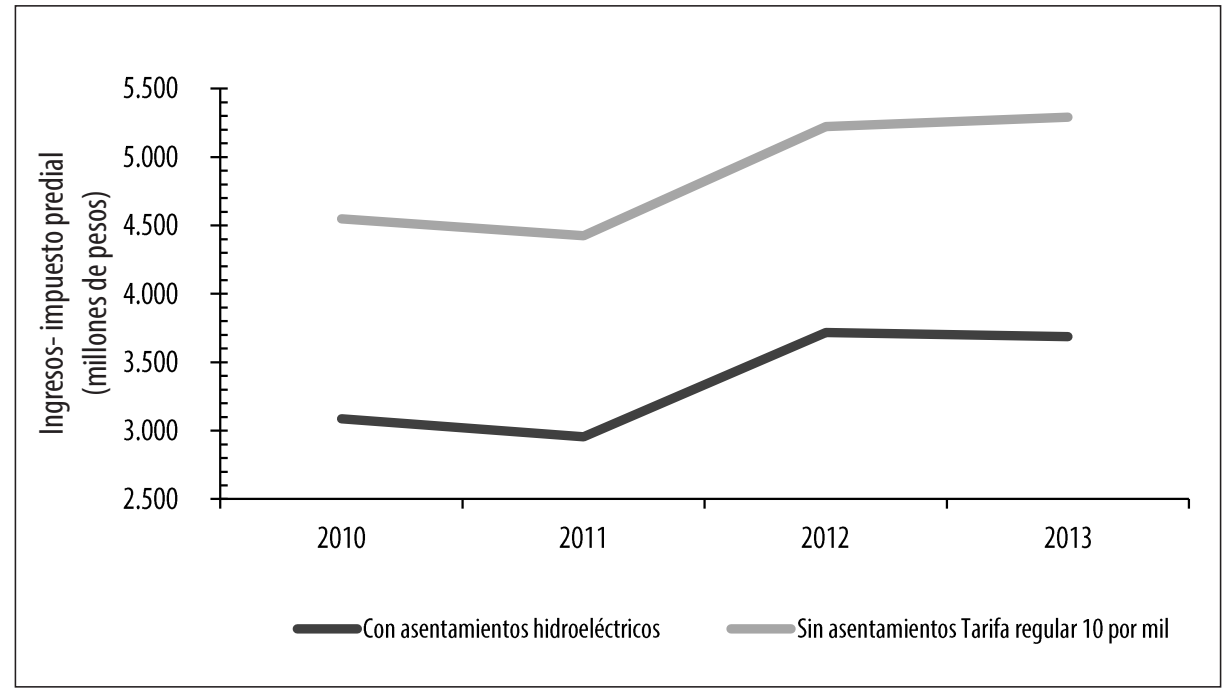

Fuente: elaboración propia con información de DNP (2014b),

\subsubsection{Fallo de asignación}

Otro elemento que contribuye al deterioro tributario es el fallo en los criterios de asignación de la compensación establecidos en la Ley 56/19815. Esto se evidencia al utilizar la relación entre el área afectada y el área total. En Guatapé, la relación es del $47,4 \%$ del área afectada, mientras que para El Peñol solo es del 24,58\%. Sin embargo, de acuerdo tabla 6, El Peñol debió recibir mayores ingresos por concepto de la compensación del impuesto predial, cuando es Guatapé el más afectado acorde a la Ley 56/1981 (ver tabla 3).

La deducción inevitable de este esquema de asignación es que entre mayor sea el número de predios afectados, el área restante, sobre el cual se determina el pago, será menor ${ }^{6}$ al igual que el avalúo ${ }^{7}$. De este criterio de asignación establecido

5 La ecuación para el cálculo viene dada por

6 Está definida como diferencia entre área total y área afectada.

7 El promedio de hectáreas afectadas por Ley 56/1981 en el 2013 para El Peñol fue de \$8,82 millones y para Guatapé fue de $\$ 8,3$ millones, pero el avalúo del resto del municipio fue de $\$ 75.733$ millones y \$33.170 millones para El Peñol y Guatapé, respectivamente. 
por la Ley resultan favorecidas las empresas generadoras, ya que por formula de compensación tanto el avaluó del resto del municipio como las tarifas resultan menores.

En este sentido, el criterio de la compensación ha fallado porque está calculado sobre valores absolutos de área afectada, en vez de cifras relativas. Por tanto, la compensación adquiere un carácter regresivo, ya que el municipio deja de percibir impuestos de los sectores tradicionales que son los que más tributan, tanto en predial como en industria y comercio, en favor de las tarifas preferenciales que se le han concedido al sector eléctrico. Lo cual reduce la recaudación total del municipio, tal como se mostró en la tabla 6.

\subsection{Distorsión de la información sobre predios afectados}

La información reportada a las empresas para el pago de la compensación presenta incoherencias respecto al número de predios afectados dentro de los municipios. Esto se explica por el procedimiento llevado a cabo para el cálculo de la compensación del impuesto predial, en el cual se toman aquellos predios diferentes de los inundados. Por tal razón no se tienen en cuenta los predios afectados, que son justamente los más valorados, máxime si se tienen en cuenta los equipamientos técnicos que incorporan valor a estos predios. Así, la tarifa media de liquidación del impuesto es más baja, dado el añejamiento del avalúo catastral.

También, se ha advertido la presencia de alteraciones en el número de predios afectados, tanto por mal reporte de las empresas generadoras (omisiones de información) como por asuntos de delimitación catastral. El efecto fiscal termina en la reducción del monto compensatorio. Se observó entonces, para el caso de los municipios de estudio, que tres municipios presentan disminución en las hectáreas afectadas $^{8}$, a pesar de que un predio constitutivo de una obra no puede ser sacado de la información catastral a menos que el proyecto haya terminado, lo cual no ha sucedido en estos municipios.

Este es el caso de San Carlos al cual se le reporta un total de 197 has afectadas en 2013; no obstante que para el 2008 contaba con 269 has de acuerdo con la Resolución 686/1994. Para la sola central de Punchiná ISAGEN (2012) se registraba 366 has afectadas y cuatro has en la de Calderas. De igual manera, El Peñol pasa de 2.848 a 2.798 has afectadas -Resolución 686/1994- entre el 2008 y 2013; en igual periodo, San Roque pasa de 658 a 612 has afectadas. Esta situación junto con las

8 Información obtenida a partir de las resoluciones catastrales emitidas por la dirección de sistemas de Información y Catastro departamental de Antioquia. 
Efectos fiscales de los asentamientos hidroeléctricos: el caso de la cuenca de los ríos Negro y Nare en Colombia

tarifas preferenciales a las generadoras magnífica el detrimento fiscal de los municipios por efecto de la ausencia de claridad en la información con la que se liquida la compensación

Así mismo, el reporte de la información, sobre la cual se debe calcular la compensación, no es preciso, particularmente cuando operan dos empresas hidroeléctricas en el mismo municipio, ya que no existen reportes por hectáreas afectadas correspondientes a cada empresa. Esta ambigüedad la presentan San Carlos, Granada, Alejandría y San Rafael; lo cual permite inferir que las empresas generadoras son auto-liquidadoras, en razón a que ellas mismas reportan los predios adquiridos y sus usos, sin respaldo de un ente catastral que verifique la información.

Resulta entonces que si la mayor valorización comercial del mercado de tierras, que se presenta con la sola declaratoria de utilidad pública de un asentamiento hidroeléctrico, mejora el valor de la base tributaria; no es coherente que, desde lo fiscal, se evidencie un debilitamiento de la recaudación fiscal. En consecuencia, los AH carecen de un enfoque territorial, porque no solo dejan de compensar a los municipios por los sacrificios de la base productiva, sino también porque los despojan de las rentas fiscales.

\section{CONCLUSIONES Y RECOMENDACIONES}

El impuesto predial es el instrumento de política tributaria más apto para introducir eficiencia y equidad en el financiamiento de los bienes públicos locales; por tal razón es indudable que las exenciones del predial y la congelación en las tarifas privilegian, en el contexto regional, al sector de más alta productividad, que es el sector eléctrico. Este sector obtiene ganancias extraordinarias dado que renta sobre el capital natural de los municipios a costo cero, y además porque capta rentas derivadas del mayor valor que le confieren a las propiedades una explotación altamente productiva y unos avalúos catastrales que distan mucho del valor comercial.

En efecto, se hace necesario eliminar el rezago tarifario y las preferencias en los avalúos para el sector eléctrico, establecidos en el Artículo 4to de la Ley 56/1981. Esto permitiría eliminar la regresividad tributaria que suponen tanto la congelación de tarifas como la desactualización de los avalúos de las tierras dedicadas a la generación de hidroelectricidad, a la vez que exigiría a las empresas involucradas pagar tarifas acordes con las rentabilidades.

Por la anterior condición, las tasas impositivas para el sector eléctrico deberían ser ascendentes y progresivas, además de incluyentes. Situación que supone no solo gravar los espejos de agua de los embalses que inundan grandes extensiones de 
tierras locales, sino también tomar en cuenta la recomendación de la Ley 1607/2012, en el sentido de aplicar el Artículo 7mo de la Ley 56/1981 sobre la capacidad instalada, comercialización y generación en un solo hecho gravable.

Para superar sesgos de información y evitar que las empresas generadoras se conviertan en autoliquidadores de los impuestos compensatorios, y aún de las transferencias, es necesario modernizar el catastro para efectos de delimitar cada propiedad hidroeléctrica a través de un plano catastral. Esto evitaría problemas de liquidación de las compensaciones del predial y evitaría rezagos en los montos tributarios por desactualización de los avalúos. De esta manera, los municipios tendrían un respaldo en la información utilizada para llevar a cabo el seguimiento a las compensaciones fiscales.

De otra parte, se hace necesario un replanteamiento en la distribución regional de las rentas del sector eléctrico, ya que los propietarios de las obras hidroeléctricas absorben la mayor parte de las rentas, en razón de la propiedad de la infraestructura técnica y en perjuicio del patrimonio hidrológico que aportan las regiones.

Esta situación del sector eléctrico se ve justificada por Piketty (2014) citado por Uribe (2014) con el siguiente argumento: "Cuando la tasa de crecimiento de la rentabilidad del capital supera la tasa de crecimiento del producto y el ingreso, [...] el capitalismo genera desigualdades arbitrarias e insostenibles que socavan radicalmente los valores meritocráticos en los que se basan las sociedades democráticas".

El enfoque del al artículo estuvo orientado a analizar los efectos fiscales en los municipios del oriente y nordeste antioqueño, respecto a la compensación del impuesto predial establecido en la Ley 56/1981; por tal razón es necesario examinar otro tipo externalidades negativas tales como: la prolongación de los calendarios de ejecución de los proyectos y los sobrecostos originados en la valoración de riesgos asociados al crédito y la inflación. En futuras investigaciones la inclusión de estos aspectos permitiría una mejor estimación de los costos reales de los proyectos y los impactos en las finanzas municipales.

\section{BIBLIOGRAFÍA}

Acosta, Corina (2004). Efecto de las empresas transnacionales en las comunidades indígenas, Endesa y la comunidad mapuche-pehuenche. [En línea] UDLAP, México, 12 de mayo de 2004126 p. [Consultado 15 de octubre de 2014].

Alcaldía de El Peñol (2014). Historia de El Peñol. [En línea] Alcaldía de El Peñol. [Consultado 26 de mayo de 2014]. 
Efectos fiscales de los asentamientos hidroeléctricos: el caso de la cuenca de los ríos Negro y Nare en Colombia

Alcaldía de Alejandría (2004). Acuerdo No 013 del 30 de diciembre de 2004. [En línea] Alcaldía de Alejandría, Antioquia. [Consultado 20 de octubre de 2014].

Alcaldía de El Peñol (2006). Acuerdo No 016 del 30 de noviembre de 2006. [En línea] Alcaldía de El Peñol, Antioquia. [Consultado 20 de octubre de 2014].

Alcaldía de Granada (2004). Acuerdo No 15 del 5 de diciembre de 2004. [En línea] Alcaldía de Granada, Antioquia. [Consultado 20 de octubre de 2014].

Alcaldía de Guatapé (2013). Acuerdo 14 No del 30 de diciembre de 2013. [En línea] Alcaldía de Guatapé, Antioquia. [Consultado 20 de octubre de 2014].

Alcaldía de San Carlos (2005). Acuerdo No 19 de 2005. [En línea] Alcaldía de San Carlos, Antioquia. [Consultado 20 de octubre de 2014].

Alcaldía de Santo Domingo (2010). Acuerdo No 015 de 2010. [En línea] Alcaldía de Santo Domingo, Antioquia. [Consultado 20 de octubre de 2014].

Alcaldía de San Roque (2006). Acuerdo No 014 del 9 de diciembre de 2006. [En línea] Alcaldía de San Roque, Antioquia. [Consultado 20 de octubre de 2014].

Ansar, Atif; Flyvbjerg, Bent; Budzier, Alexander y Lunn, Daniel (2014). Should we build more large dams? The actual costs of hydropower megaproject development. En: Energy Policy, Vol. 69, p. $43-56$.

Aramburo Clara y Carmona Sergio (1990). Colección de Estudios de localidades-Guatapé. Medellín, CORNARE, INER, $71 \mathrm{p}$.

BIRDAntioquia (2011). Potencial hidroeléctrico de Antioquia: Inventario, perspectivas y estrategias. [En línea] Gobernación de Antioquia, Medellín, Banco de Iniciativas Regionales para el Desarrollo de Antioquia-BIRD Antioquia, Abril de 2011, 111p. [Consultado 23 de noviembre de 2014 l.

Bonet, Jaime (2006). Desequilibrios regionales en la política de descentralización en Colombia. [En línea] Centro de Estudios Económicos Regionales No 77, CEER, Bogotá, Banco de la República de Colombia, octubre de 2006, 51p. [Consultado 16 de enero de 2015].

Cárdenas, Mauricio y Mercer-Blackman, Valerie (2005). El sistema tributario colombiano: impacto sobre la eficiencia y la competitividad. [En línea] Fedesarrollo, Bogotá, Cámara de Comercio Colombo-Americana Confecámaras, 31 de agosto de 2005, 138 p. [Consultado 18 de enero de 2015].

Catastro Departamental (2013). Resolución 017 de 2013, Medellín, Departamento administrativo de Planeación, Gobernación de Antioquia.

Comisión Mundial de Represas (2000). Represas y desarrollo: un nuevo marco para la toma de decisiones. [En línea] Comisión Mundial de Represas, Reino Unido y Estados Unidos, Comisión Mundial de Represas, 2000, 404p. [Consultado 20 de enero de 2015]. 
CC- Congreso de Colombia (1981). Ley 56 de 1981. Por la cual se dictan normas sobre obras públicas de generación eléctrica, y acueductos, sistemas de regadío y otras y se regulan las expropiaciones y servidumbres de los bienes afectados por tales obras. [En línea] Diario Oficial No. 35.856, Bogotá, Congreso de la República de Colombia, 5 de octubre de 1981, 16p. [Consultado 20 de mayo de 2014].

CC- Congreso de Colombia (1982). Decreto 2024 de 1982, por el cual se reglamenta parcialmente la Ley 56 de 1981. [En línea] Diario Oficial No. 360556, Bogotá, Congreso de la República de Colombia, julio 12 de 1982.|Consultado 21 de mayo de 2014].

CC-Congreso de Colombia (1993). Ley 99 de 1993. Por la cual se crea el Ministerio del Medio Ambiente, se reordena el Sector Público encargado de la gestión y conservación del medio ambiente y los recursos naturales renovables, se organiza el Sistema Nacional Ambiental, SINA y se dictan otras disposiciones. [En línea] Diario Oficial No. 41.146, Bogotá, Congreso de la República de Colombia, 22 de diciembre de 199, 12p. [Consultado 30 de junio de 2014].

CC- Congreso de Colombia (2012). Ley 1607 de 2012, Por la cual se expiden normas en materia tributaria y se dictan otras disposiciones. [En línea] Diario Oficial No. 48.655, Bogotá, Congreso de la República de Colombia, 26 de diciembre de 2012, 30p.|Consultado 21 de abril de 2015].

DNP - Departamento Nacional de Planeación (2013). Reajuste de avalúos catastrales para la vigencia de 2014. [En línea] Consejo Nacional de Política Económica y Social-CONPES- 3792, Bogotá, Departamento Nacional de Planeación, 18 de diciembre de 2013, 15p. [Consultado 25 de septiembre de 2014].

DNP - Departamento Nacional de Planeación (2014a). Desempeño fiscal de los departamentos y municipios 2013. [En línea] Dirección de Desarrollo Territorial Sostenible Grupo de Estudios Territoriales, Bogotá, Departamento Nacional de Planeación, 206p.|Consultado 25 de enero de 2015].

DNP - Departamento Nacional de Planeación (2014b). Operaciones Efectivas de Caja. [En línea] Dirección de Desarrollo Territorial Sostenible Grupo de Estudios Territoriales, Bogotá, Departamento Nacional de Planeación. [Consultado 29 de Mayo de 2015].

Dourojeanni, Axel; Jouravlev, Andrei y Chávez, Guillermo (2002). Gestión del agua a nivel de cuencas: teoría y práctica. [En línea] División de Recursos Naturales e Infraestructura, Santiago de Chile, Comisión Económica para América Latina y el Caribe (CEPAL), agosto de 2002, 83p.|Consultado 20 de octubre de 2015].

Fuentes Bernal, Juan José (2013). Apuntes sobre el cobro del ICA a las empresas generadoras de energía. [En línea] Revista Andesco, Issue No 27, Bogotá, noviembre de 2013, Andesco, 26-46 p. [Consultado 25 de octubre de 2014].

Fuentes, Juan Alberto (2013). Panorama fiscal de América Latina y el Caribe: Reformas tributarias y renovación del pacto fiscal. [En línea] Comisión Económica para América Latina y el Caribe (CEPAL), Santiago de Chile, CEPAL, febrero de 2013, 53p.|Consultado 1 de febrero de 2015].

Gobernación de Antioquia (1985). Guía para la aplicación de la ley 56/1981. Medellín, Gobernación de Antioquia, 48p. 
Efectos fiscales de los asentamientos hidroeléctricos: el caso de la cuenca de los ríos Negro y Nare en Colombia

Gobernación de Antioquia (1994). Resolución Número 686 de 1994. Medellín, Gobernación de Antioquia.

Guhl, Ernesto (2014). Territorios sostenibles: una opción frente a la crisis ambiental. [En línea] Razón Pública, Bogotá, 24 de marzo de 2014. [Consultado 23 de mayo de 2015].

Iregui, Ana; Melo, Ligia y Ramos, Jorge (2004). El impuesto predial en Colombia: Factores explicativos del recaudo. [En línea] Subgerencia Estudios Económicos, Bogotá, Banco de la República, octubre de 2004, 38 p. [Consultado 29 de enero de 2015].

ISAGEN (2012). Transferencias de ley del sector eléctrico. [En línea] ISAGEN, Bogotá, ISAGEN, noviembre de 2012, p. 10.|Consultado 18 de julio de 2014].

Jorratt, Michael (2010). Metodología para medir el impacto fiscal de los gastos tributarios subnacionales en Colombia. [En línea] Sector de Capacidad Institucional y Finanzas, Washington, D.C., Banco Interamericano de Desarrollo, junio de 2010, p. 43. [Consultado 18 de julio de 2014].

Larrahondo, Miryam (1993). Aprovechamiento acuicola de embalses en Colombia en Anvances en el manejo en Aprovechamiento acuicola de embalses en América Latina y el Caribe. [En líneal Organizacion de las Naciones Unidas para la Agricultura y la Alimentacion - FAO, México D.F, FAO, junio de 1993, 163p. [Consultado 18 de julio de 2015].

Martínez, Viviana (2013). Continuidades y disrupciones de los condicionantes de los conflictos ecológico-distributivos por las hidroeléctricas en Colombia. [En línea] VI Jornadas de la Asociación Argentino Uruguaya de Economía Ecológica, Salta, Argentina, Universidad Nacional de Salta, 26 de noviembre de 2013, 18p. [Consultado 12 de mayo de 2015].

Naranjo, Alberto; Muñoz, Juan; Mesa, Manuel y Londoño Sebastían (2011). El impuesto predial municipal en Antioquia: Hacia el diseño de un recaudo óptimo, [En línea] Universidad EAFIT, Medellín, Gobernación de Antioquia, 111p.|Consultado 1 de junio de 2015].

Nuñez, Jairo (2005). Diagnóstico básico de la situación de los ingresos por impuestos del orden municipal en Colombia. [En línea] Centro de Estudios sobre Desarrollo Económico, Bogotá, Universidad de los Andes, 138p.|Consultado 14 de mayo de 2015].

OCDE y CEPAL (2014). Evaluaciones del desempeño ambiental - Colombia. [En línea] Organización para la Cooperación y el Desarrollo Económicos, OCDE-CEPAL, abril de 2014, 258p. Consultado 20 de septiembre de 2014].

Piketty, Thomas (2014). Capital in the Twenty-First Century. Cambridge: The Belknap, Cambridge, Press of Harvard University Press, 696p.

PROESA (2013). Estudio de impacto ambiental del proyecto Aprovechamiento hidroeléctrico del río Samaná Norte. [En línea] Cornare, Medellín, PROE S.A.S. E.S.P. [Consultado 20 octubre de 2015].

Torres, Maria Adelaida (2013). Análisis de nuevas dinámicas territoriales por proyectos de infraestructura y su influencia en la generación de conflictos socio ambientales. Caso de estudio: 
Hidroituango. [En línea] Universidad Nacional de Colombia, sede Medellín, Medellín, 164p. [Consultado 19 de octubre de 2015].

Uribe, Mauricio (2014). Desigualdad versus Democracia. [En línea] Semanario Virtual Caja de Herramienta edición No 00401, Bogotá, 30 de mayo de 2014. [Consultado 30 de junio de 2014].

Vélez, Angela; Arroyave, Guillermo; Giraldo, Hector y Vélez, Luis Diego (1982). Impactos regionales de los proyectos Hidroeléctricos. Medellín, Departamento Administrativo de Planeación, Gobernación de Antioquia, 206p.

XM (2014). Informe Ejecutivo. [En línea] XM S.A E.S.P, Medellín, XM, 6 de noviembre, 7 p. [Consultado 29 de junio de 2014]. 